\title{
Collagen fiber changes related to keratoconus with secondary corneal amyloidosis
}

This article was published in the following Dove Press journal: International Medical Case Reports Journal

\author{
Kaoru Araki-Sasaki' \\ Yasuhiro Osakabe ${ }^{2}$ \\ Koji Fujita ${ }^{2}$ \\ Kazunori Miyata ${ }^{3}$ \\ Koji Hirano ${ }^{4}$
}

'Department of Ophthalmology, Japan Community Health Care Organization, Hoshigaoka Medical Center, Hirakata, Japan; ${ }^{2}$ Department of Molecular Pathology, Tokyo Medical University, Tokyo, Japan; ${ }^{3}$ Department of Ophthalmology, Miyata Eye Hospital, Miyakonojo, Japan; ${ }^{4}$ Department of Ophthalmology, Ban Buntane Hotokukai Hospital, Fujita Health University, Nagoya, Japan
Correspondence: Kaoru Araki-Sasaki Department of Ophthalmology, Japan Community Health Care Organization, Hoshigoaka Medical Center, 4-8-I, Hoshigaoka, Hirakata, Osaka 57385II, Japan

Tel $+8|72840264|$

Fax+8I 728402266

Email sasakis@sa2.so-net.ne.jp

\begin{abstract}
We describe the histological changes in the collagen fibers of a 50-year-old male who presented keratoconus with secondary corneal amyloidosis. Corneal tissue from the patient was obtained following a penetrating keratoplasty and was subjected to histochemical analysis using Masson's trichrome staining, Congo red staining, anti-lactoferrin antibody, and anti-transforming growth factor-beta-induced protein (TGFBIp) antibody. A Congo red-positive region was detected in the anterior half of the stroma in the center and inferior cornea. Although hemotoxylin and eosin staining revealed irregularity in the Congo red-positive region, other parts of the stroma did not show any abnormalities. Positive staining both by anti-TGFBIp and anti-lactoferrin antibodies was observed in the Congo red-positive region. Interestingly, all the layers of the corneal stroma, including the peripheral region, were positively stained by anti-TFGBIp antibody, even in the Congo red-negative area. Masson's trichrome staining also showed irregular staining throughout the corneal stroma, even outside of the Congo redpositive region. Additionally, Bowman's layer, which consists of collagen type IV, was damaged. TGFBIp was strongly expressed and Masson's trichrome staining was reduced throughout the entire keratoconic stroma. The constant qualitative changes in keratoconic collagen fibers, along with the observed abnormality in the Bowman's membrane, might point to the pathogenesis of secondary corneal amyloidosis in keratoconus.
\end{abstract}

Keywords: cornea, amyloid, stroma, TGFBIp, lactoferrin

\section{Introduction}

The pathogenesis of keratoconus is still unclear. Previous studies using histochemical and biochemical analysis have pointed to a variety of possible causes for keratoconus, such as the upregulation of various enzymes, excessive keratocyte apoptosis induced by the interleukin-1 system, abnormal distribution of collagen orientation, and abnormal cytokine expression. ${ }^{1-3}$ Recently, collagen abnormalities have been reported to be directly associated with keratoconus by studies using multiple methods. ${ }^{4,5}$

Clinically, we often observe stromal edema following lamellar keratoplasty in keratoconus patients, and the edema in such cases is more obvious than that observed in leukoma patients. Additionally, we observe that the suture is easy to loosen following keratoplasty for keratoconus. These observations led us to speculate that the stromal component might be qualitatively altered in keratoconus. Thus, the keratoconic corneal stroma might be affected by an abnormality other than a genetic mutation within TGFBI as was reported. ${ }^{6}$

Secondary corneal amyloidosis (SCA) occurs with progressive keratoconus at a frequency ranging from $3.5 \%$ to $17.6 \% .^{7,8}$ This indicates that protein aggregation 
tends to increase in the keratoconic corneal stroma. Previously, we reported that SCA in trichiasis is predominantly induced by lactoferrin Glu561 Asp polymorphism. ${ }^{9}$ A report by Nisson and Dobson ${ }^{10}$ suggests that Glu561 Asp lactoferrin might aggregate and result in amyloidosis. However, we did not observe this polymorphism in SCA in keratoconus. ${ }^{11}$ Therefore, we speculate that another amyloidogenic protein might play a key role in SCA in keratoconus.

In 2009, Tai et $\mathrm{al}^{7}$ reported that transforming growth factor-beta-induced protein (TGFBIp) is present in the corneal stroma of patients with keratoconus. Subsequently, TGFBIp has been shown to form the core in amyloidosis due to the high number of beta-sheets within the structure of this protein. ${ }^{12-14}$

In this study, we examined TGFBIp and lactoferrin expression in a keratoconus patient with SCA using anti-TGFBIp and anti-lactoferrin antibodies. We also used Masson's trichrome staining to detect the native collagen, and we observed the affected collagen in the cornea with keratoconus.

\section{Case}

The protocol used in this report was approved by the Institutional Review Board of the Japan Health Care Organization Hoshigaoka Medical Center. Written informed consent including publication of the case details and accompanying images was obtained from the patient, and the procedures used conformed to the tenets of the Declaration of Helsinki. A 50 -year-old male who had suffered from keratoconus for $>40$ years complained of progressive opacity in his right cornea. The corrected visual acuity of his right eye was 20/1000. He also presented with atopic skin disease. Slit-lamp examination revealed a milky white soft mass on the top of the keratoconic cornea (Figure 1A). In accordance with our previously published classification method for $\mathrm{SCA},{ }^{15}$ the mass was classified as having a gelatinous drop-like dystrophy-type appearance. The patient had complained of a foreign body sensation, epiphora, and redness of his right eye. Mild vessel invasion toward the white mass was observed.

Although the cornea of the fellow eye protruded, which is a typical finding of keratoconus, a milky white mass was not observed. Instead, slit-lamp examination revealed that the fellow cornea showed a linear subepithelial opacity, which was speculated to be an early stage of SCA (Figure 1B). ${ }^{16}$

To resolve the symptoms and reduced visual acuity caused by the white mass on his cornea, the patient underwent a penetrating keratoplasty (PKP) on his right eye. Histochemical analysis was performed for diagnosis. After the excised specimen of the PKP was fixed with $4 \%$ paraformaldehyde at $4^{\circ} \mathrm{C}$, $3 \mu \mathrm{m}$ sections were cut and mounted on slides. After the slides had dried, samples were stained with Congo red, Masson's trichrome, and hemotoxylin and eosin (H\&E). Staining with anti-lactoferrin antibody (Proteintech, Chicago, IL, USA) and anti-TGFBIp/BIG3 antibody (Proteintech) was also performed.

All the sections were incubated with 1\% bovine serum albumin in $\mathrm{PBS}$ at $24^{\circ} \mathrm{C}$ for 10 minutes each in order to block any nonspecific binding. Subsequently, the samples were incubated with anti-lactoferrin antibody and anti-TGFBI/ BIG3 antibody (described above) for 90 minutes at room temperature. The sections were then washed three times in PBS for 10 minutes, and the binding of the antibodies was followed by a reaction with biotinylated goat anti-rabbit immunoglobulin $\mathrm{G}$ and horseradish peroxidase-conjugated streptavidin. The slides were dehydrated using an ethanol series (70\%-95\%) and xylene, and were then covered with a coverslip using the mounting medium (Malinol; Muto Pure Chemicals Co. Ltd, Tokyo, Japan). All slides were examined by both light and polarizing microscopy (Leica DM4000 B; Leica Microsystems, Wetzlar, Germany).

Figure 2A shows the results of Congo red staining of the excised cornea. Although the superior peripheral stroma
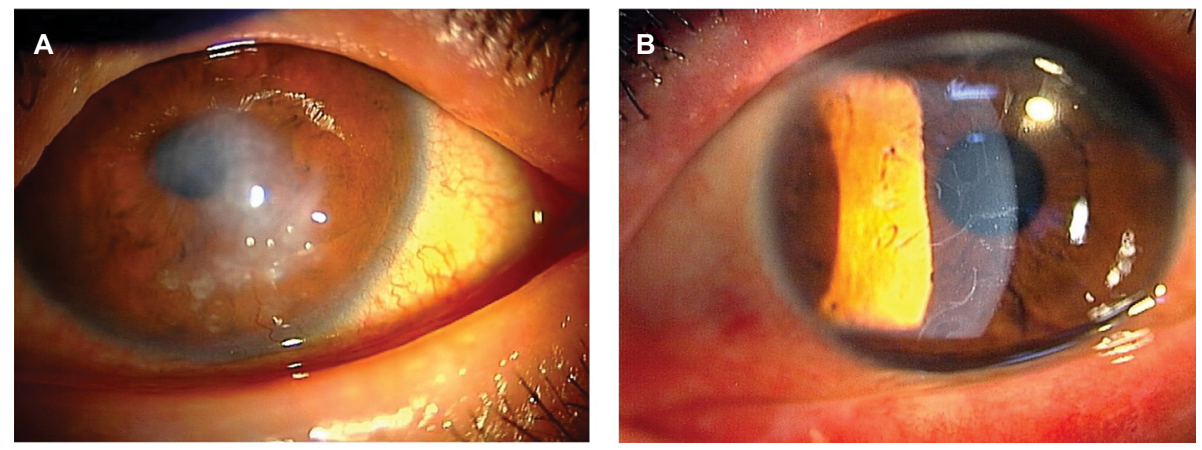

Figure I Preoperative slit-lamp photographs.

Notes: (A) Slit-lamp photograph of the right eye before surgery. A milky white mass on the tip of the keratoconic cornea was observed. (B) Slit-lamp photograph of the patient's fellow eye. The keratoconic cornea was observed, and linear opacity under the epithelium was noted instead of a milky white mass. 
did not show any marked changes (Figure 2B), Congo red staining was detected in the central and inferior peripheral stroma (Figure 2C), near the limbus. Figure 3A shows the results of $\mathrm{H} \& \mathrm{E}$ staining of the collagen fibers in the cornea. Although the superior peripheral stroma did not show any abnormal staining (Figure 3B), irregularity was observed in the center of the cornea (Figure 3C), in the same region where the amyloid was detected by Congo red staining (Figure 2).

Figure 4 shows the results of Congo red (Figure 4A, B), anti-lactoferrin antibody (Figure 4C, D), and anti-TGFBIp antibody (Figure 4E, F) staining. The eosinophilic material was positively stained with Congo red (Figure 4A, B). This material was mainly located in the stroma as well as in the epithelial layers that showed destruction of Bowman's layer. The eosinophilic material also showed a positive reaction with the anti-lactoferrin antibody (Figure 4C, D) in almost the same area as that stained positively by Congo red. In contrast, the complete corneal stroma was positively stained with the anti-TGFBIp antibody, including the Congo red-positive and -negative areas. Although staining with anti-TGFBIp antibody was stronger within the Congo red-positive stromal region, this staining was also confirmed within the Congo red-negative region (Figure 4F, arrow).
Masson's trichrome staining (Figure 5A) showed irregular staining in the central stroma. The staining was not uniform and was negative in various places in the peripheral cornea (Figure 5B, arrows) where H\&E staining showed no marked changes (Figure 3B). The staining was irregular even in the non-amyloid region (Figure 5C, arrows).

Bowman's layer, which consists of type IV collagen, was also affected by the keratoconus (Figure 6A). These changes included attenuation (Figure 6B), as well as interruption (Figure 6C) and complete disappearance (Figure 6D), when compared to the normal cornea (Figure 6E). One month after undergoing PKP, the corrected visual acuity of the patient's right eye was limited to 20/200 owing to amblyopia.

\section{Discussion}

The current report describes the first documented case of abnormal stromal Masson's trichrome staining in keratoconus with SCA. It has previously been reported that corneal stromal collagen differs in cases of keratoconus compared to normal corneas. ${ }^{1-5}$ In this study, we observed changes in collagen in keratoconus using the simple method of Masson's trichrome staining. The mechanism of Masson's trichrome staining follows a simple procedure, wherein the nucleus is
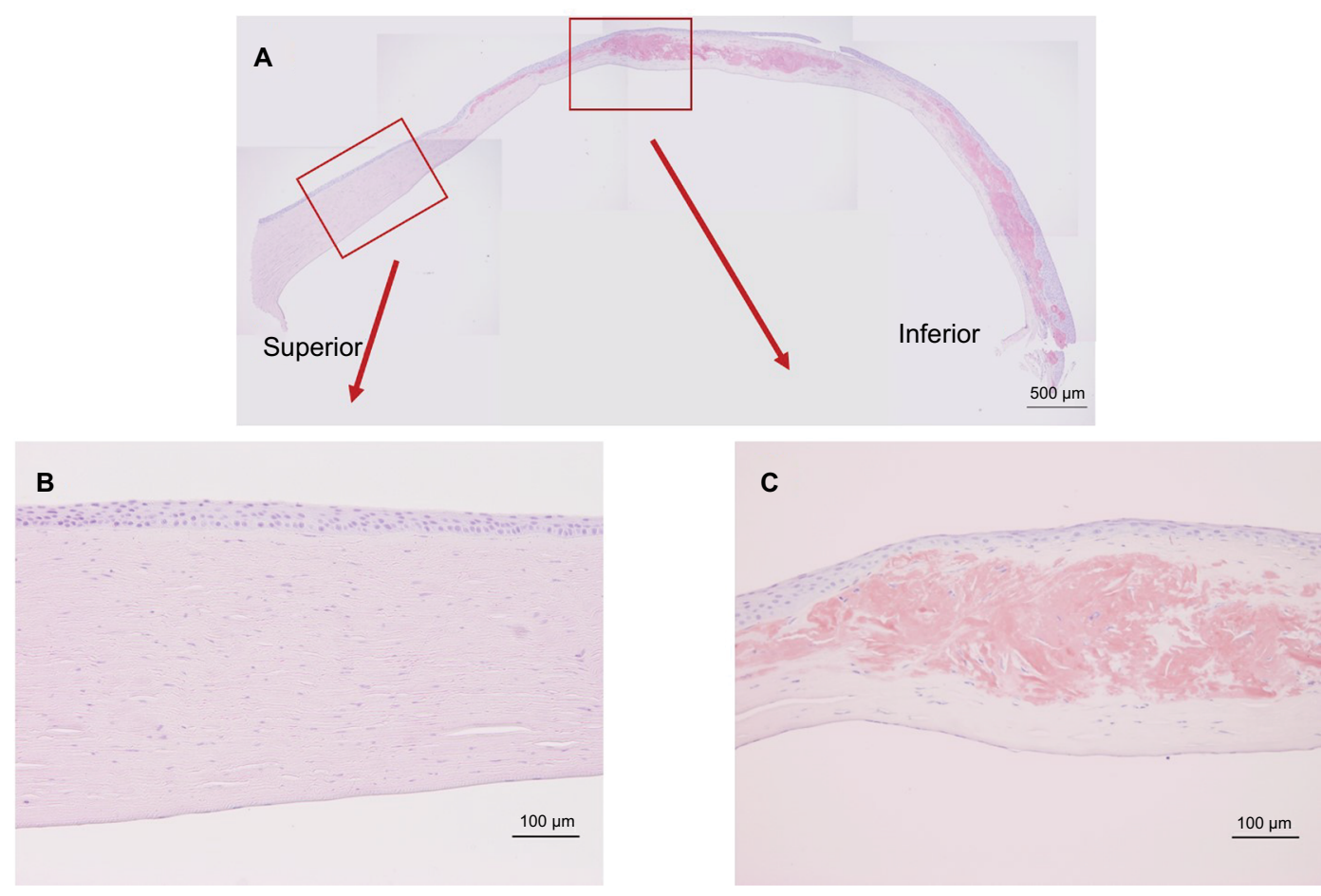

Figure 2 Congo red staining of the excised cornea.

Notes: (A) A positive stained mass was observed in the stroma connected to the inferior peripheral side of the cornea. (B) Inlet showing an enlarged view of the noted area (red arrow). The corneal stroma was normal, and no remarkable pathogenic appearance was observed in the superior peripheral corneal stroma. (C) Inlet showing an enlarged view of the noted area. The Congo red-positive mass resulted in staining in the upper half of the corneal stroma. 

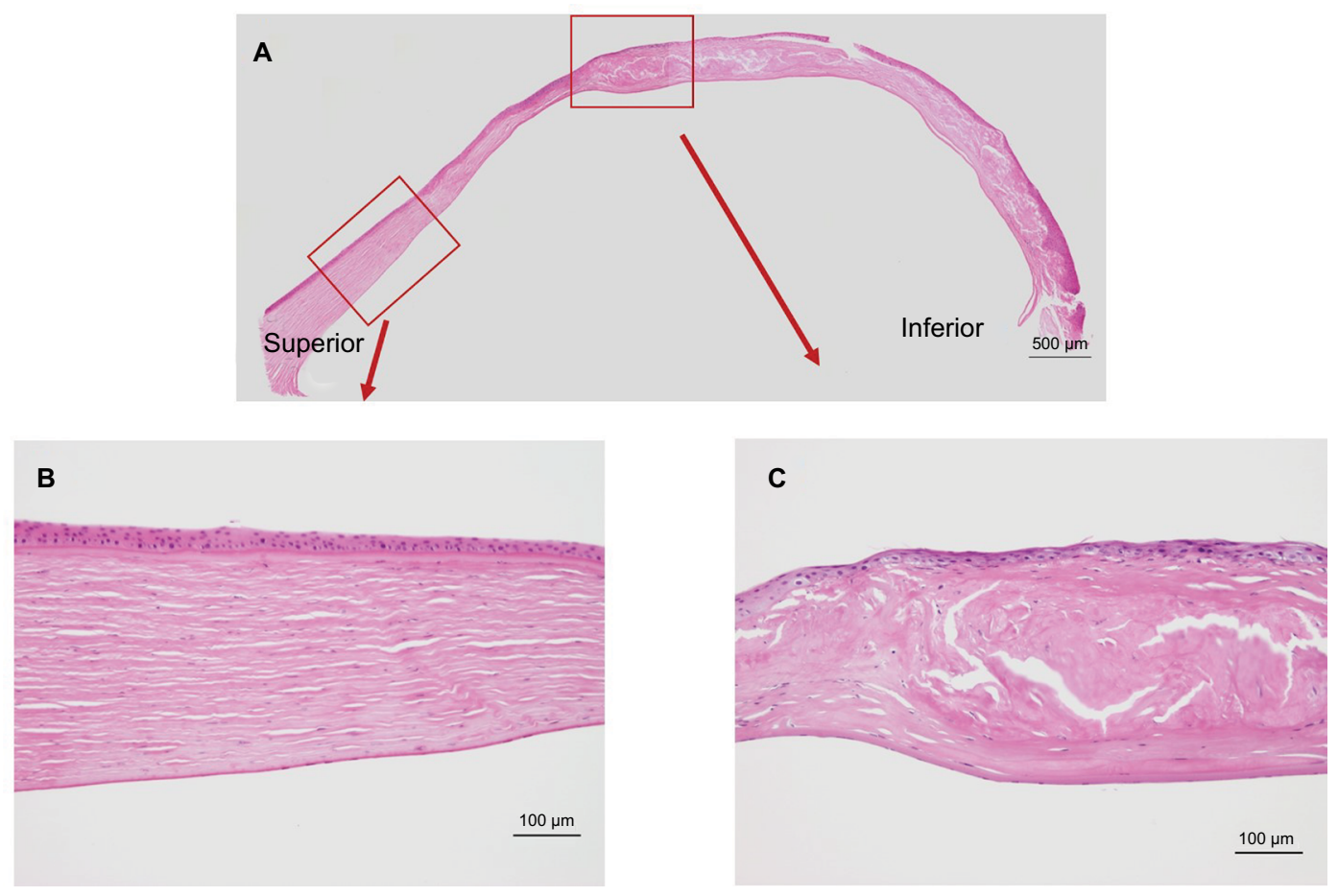

Figure 3 Hemotoxylin and eosin staining of the excised cornea.

Notes: (A) Irregularity of the collagen fibers was observed in the center of the cornea, in contrast to the regular alignment observed in the superior peripheral cornea. (B) Inlet showing an enlarged view of the peripheral cornea. (C) Inlet showing an enlarged view of the center of the cornea.

stained purple by iron hematoxylin, the cytoplasm is stained red by acid fuchsin, and the collagen is stained blue by aniline blue. This method is effective even for type I and type IV collagen staining. As can be seen in Figure 6E, normal corneal stroma was stained uniformly blue by Masson's trichrome staining. In contrast, the keratoconic cornea was not stained with Masson's trichrome staining, but was uniformly stained with anti-TGFBIp antibody.

Upregulation of various enzymes, such as lysosomal enzymes, and excessive keratocyte apoptosis by several inflammatory cytokines have been reported in keratoconus. Additionally, direct evidence for collagen fiber change has been the differing distribution of collagen orientation as observed by the synchrotron X-ray scattering pattern ${ }^{4}$ and the quantitative analysis of collagen lamellae in keratoconus, as observed by harmonic generation imaging microscopy. ${ }^{5}$ Marcatelli et al ${ }^{17}$ reported that the orientation of sutural lamellae close to Bowman's membrane is significantly different between healthy and keratoconic samples. This suggests that the collagen structure in the keratoconic cornea differs greatly from that in the normal cornea. Our observations strongly support these previous data. In our study, TGFBIp was detected diffusely throughout the whole stromal region, including the non-amyloid region. Our observation of negative Masson's trichrome staining within the non-amyloidogenic region implies that the corneal collagen in keratoconus might be altered throughout the whole stromal region, rather than in the amyloidogenic region only.

TGFBIp is produced during corneal wound healing, and has been reported to have the ability to form the core of amyloidosis because it has a large number of beta-sheets. ${ }^{12,18}$ The reason behind the aggregation of excessive amounts of TGFBIp that sometimes occurs in keratoconus remains unknown. Even with the high number of beta-sheets, a biochemical change, such as gene transformation, or changes in proteolytic enzyme levels might be necessary. At present, there is some evidence that proteins are altered in keratoconus. The upregulation of matrix metalloproteinase-9, tumor necrosis factor- $\alpha$, and interleukin- 6 has been well established in keratoconus. ${ }^{19}$ Temperature and $\mathrm{pH}$ can also accelerate protein aggregation, ${ }^{13}$ and any of these factors could potentially cause excessive TGFBIp aggregation.

It has been previously reported that SCA with trichiasis is predominantly induced by the lactoferrin Glu561Asp polymorphism, which can aggregate easily to form the core of SCA. ${ }^{10}$ However, this polymorphism is only significant in the secondary amyloidosis in trichiasis, but not in keratoconus. ${ }^{11}$ The constant expression of TGFBIp and enzymatic changes observed in keratoconus might explain this discrepancy. This further establishes the fact that the pathological mechanism 


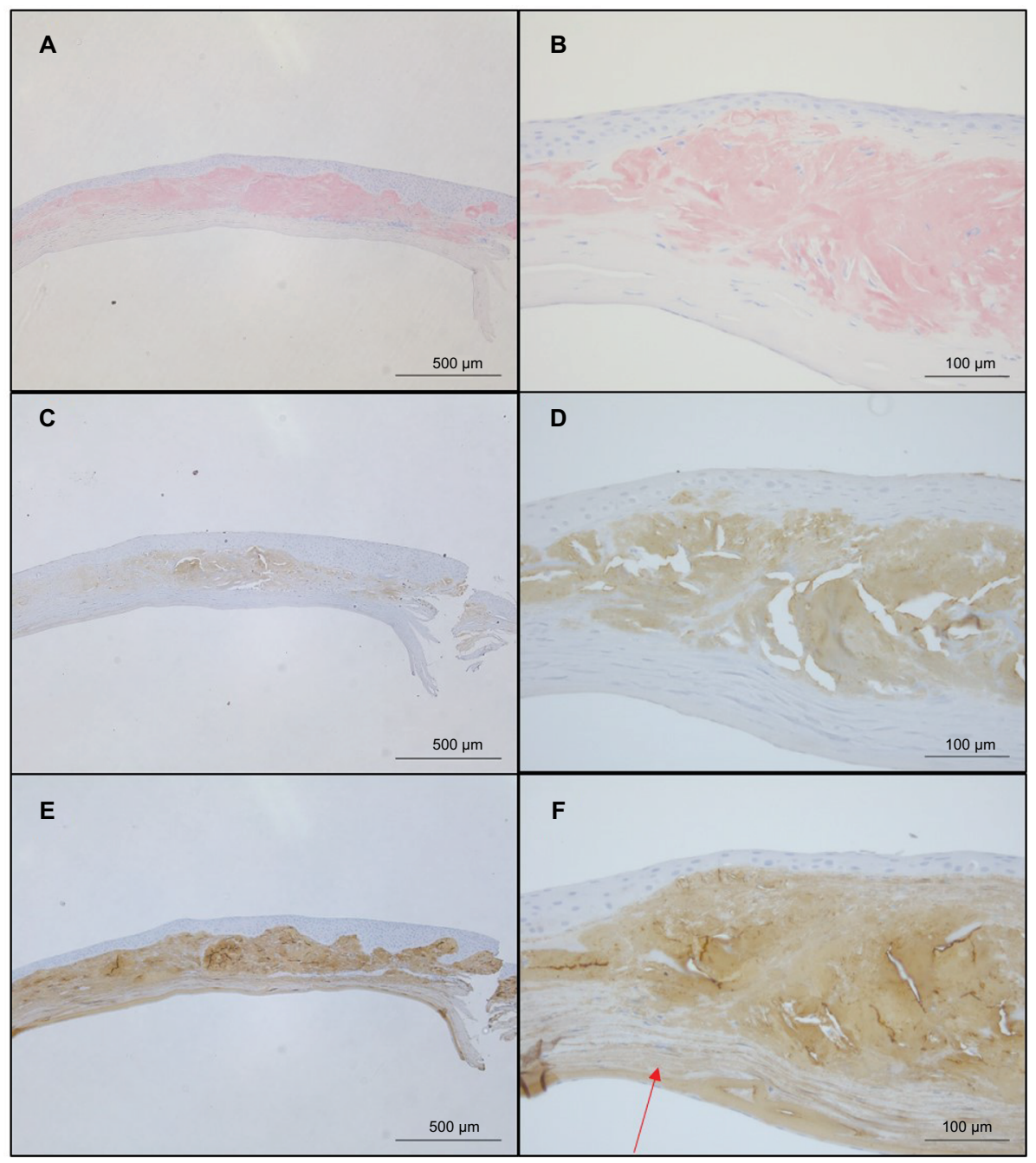

Figure 4 Comparison of staining by Congo red, anti-lactoferrin antibody, and anti-TGFBlp antibody.

Notes: (A, B) The eosinophilic material was positively stained with Congo red. (C, D) The eosinophilic material was also positively stained with anti-lactoferrin antibody in an area almost overlapping the Congo red-positive region. (E, F). The complete corneal stroma was positively stained by anti-TGFBlp antibody, including the Congo rednegative (arrow) as well as the Congo red-positive area.

of SCA in keratoconus might differ from that of SCA in trichiasis.

In our case, lactoferrin was positively stained only in the Congo red-positive region of the keratoconic cornea. Previously, we had employed ultrahigh-resolution optical coherence tomography to demonstrate that the amyloidosis in SCA caused by trichiasis deposited above the Bowman's membrane, without destroying it. Additionally, we had shown the existence of an association between lactoferrin in the tear film and SCA. ${ }^{16}$ Therefore, in the case of keratoconus, we hypothesize that lactoferrin only deposits on the core of the SCA, which is formed by other amyloidogenic proteins. We have previously proposed a classification method for SCA and have shown that patients with gelatinous drop-like dystrophy-type SCA are younger and predominantly women.
Additionally, we have reported that those who suffer from diseases associated with SCA for longer time periods are more likely to develop gelatinous drop-like dystrophy-type SCA. ${ }^{15}$ In accordance with this finding, the present report implies that SCA of gelatinous drop-like dystrophy-type, as observed in our patient, might result from modification by the gradual deposition of lactoferrin to the core amyloid region over a long period of time.

It would be advantageous to investigate the early stages of SCA in keratoconus in order to determine the mechanism of amyloid formation within the keratoconic cornea. However, histochemical analysis of early-stage keratoconus is usually prohibited by ethical considerations. Interestingly, the linear line observed in the fellow eye of our patient could be an early sign of SCA. ${ }^{20,21}$ The continuous observation of this clinical 


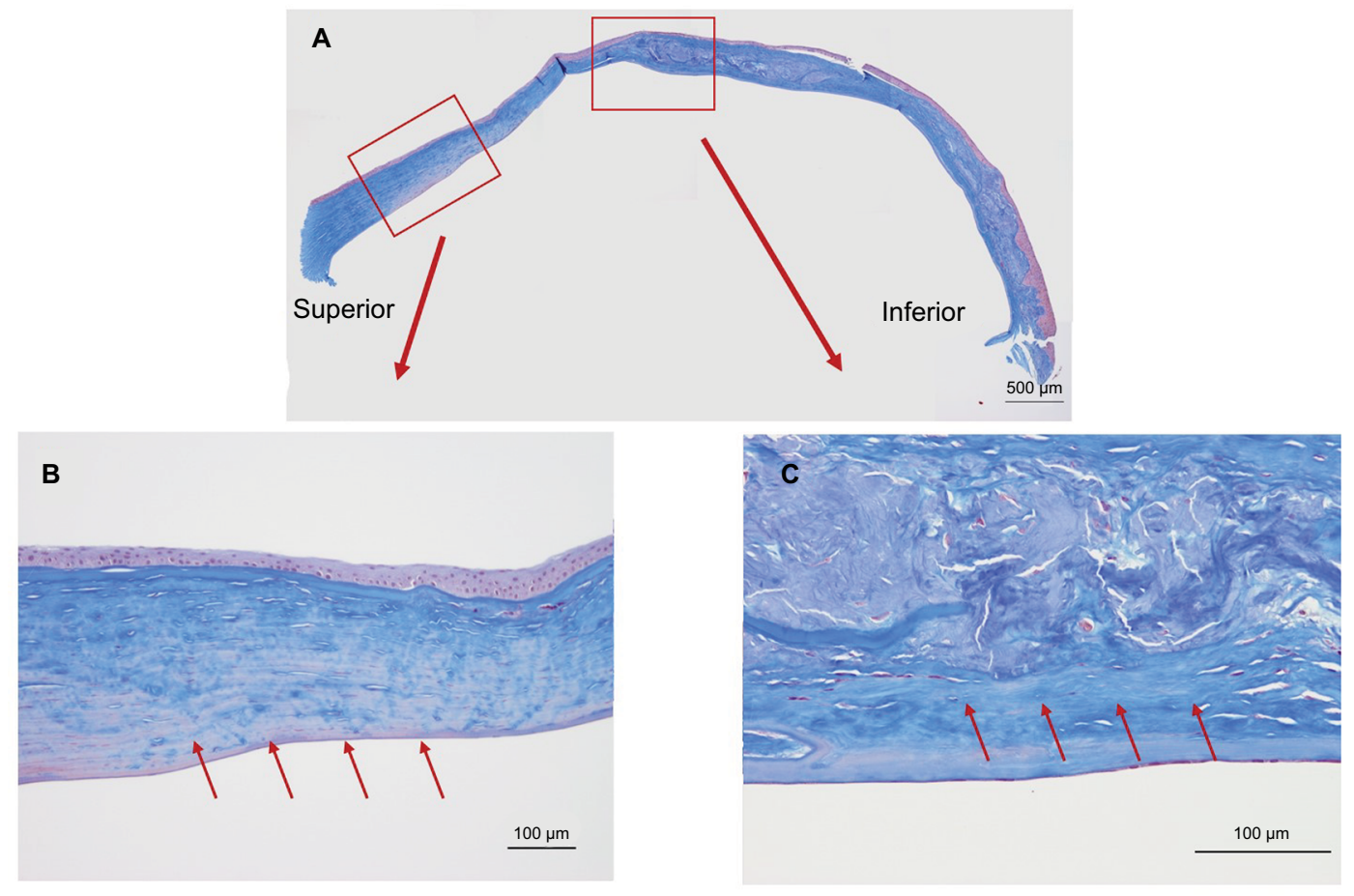

Figure 5 Masson's trichrome staining of the excised cornea.

Notes: (A) The excised cornea showed irregular collagen staining (collagen is stained blue). (B) An enlarged view of the superior peripheral cornea. (C) Higher magnification image of the central cornea. The staining was not uniform throughout the cornea and was negative at certain places within the peripheral cornea (B, arrows) as well as in the central cornea (C, arrows).

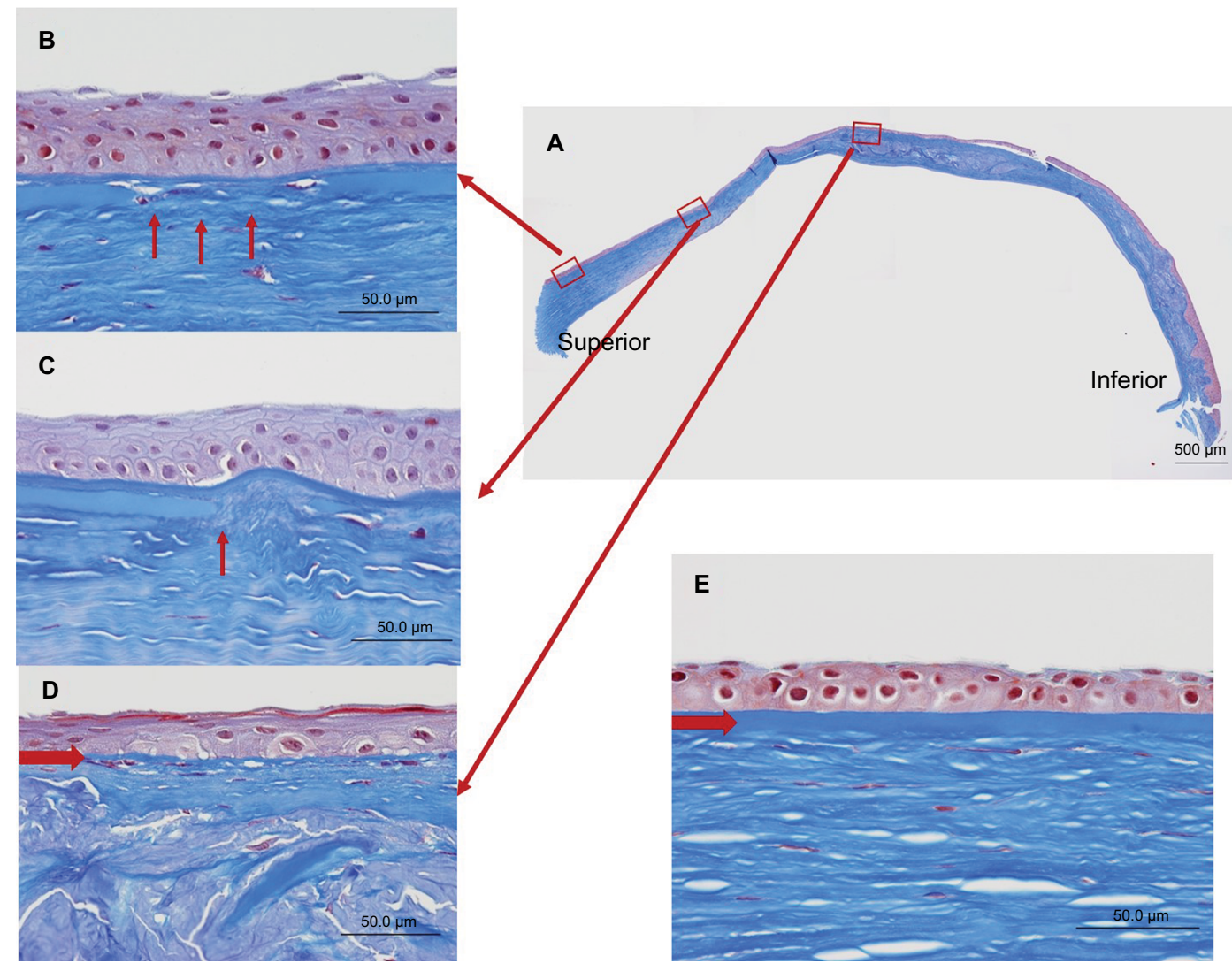

Figure 6 Masson's trichrome staining of the Bowman's layer.

Notes: (A) Staining of the excised cornea. (B-D) An enlarged view of each respective inlet. These inlets provide examples of (B) thinning (arrows), (C) cuts (arrow), and (D) complete disappearance (arrow). (E) An image of a normal corneal region for comparison. An arrow indicates Bowman's layer. 
phenomenon may, therefore, help to elucidate the mechanism of amyloid formation in keratoconus in the future.

\section{Conclusion}

In conclusion, we report a case that suggests that TGFBIp is consistently upregulated and the collagen fibers are qualitatively changed in the complete stroma of the keratoconic cornea. These changes might be a potential biomarker for determining the pathogenesis of SCA in keratoconus.

\section{Acknowledgment}

We would like to thank Editage Co. LTd. for English language editing.

\section{Author contributions}

All authors contributed toward data analysis, drafting and revising the paper and agree to be accountable for all aspects of the work.

\section{Disclosure}

The authors report no conflicts of interest in this work.

\section{References}

1. Davidson AE, Hayes S, Jardcastle AJ, Tuft SJ. The pathogenesis of keratoconus. Eye. 2014;28(2):189-195.

2. Galvis V, Sherwin t, Tello A, Merayo J, Barrera R, Acera A. Keratoconus: an inflammatory disorder? Eye. 2015;29(7):843-859.

3. Wisse RP, Kuiper JJ, Gans R, Imhof S, Radstake TR, Van der Lelij A. Cytokine expression in keratoconus and its corneal microenvironment: a systematic Review. Ocul Surf. 2015;13(4):272-283.

4. Meek KM, Tuft SJ, Huang Y, et al. Changes in collagen orientation and distribution in keratoconus corneas. Invest Ophthalmol Vis Sci. 2005;46(6):1948-1956.

5. Morishige N, Shin-Gyou-Uchi R, Azumi H, et al. Quantitative analysis of collagen lamellae in the normal and keratoconic human cornea by second harmonic generation imaging microscopy. Invest Ophthalmol Vis Sci. 2014;55(12):8377-8385.

6. Udar N, Kenney MC, Cbalukya M, et al. Keratoconus--no association with the transforming growth factor beta-induced gene in a cohort of American patients. Cornea. 2004;23(1):13-17.
7. Tai TY, Damani MR, Vo R, et al. Keratoconus associated with corneal stromal amyloid deposition containing TGFBIp. Cornea. 2009;28(5):589-593.

8. Trikha S, Sahu D, Jeffry M, Boase D. Secondary corneal amyloidosis in keratoconus. Cornea. 2011;30(6):716-717.

9. Araki-Sasaki K, Ando Y, Kitagawa K, et al. Lactoferrin Glu561 Asp facilitates secondary amyloidosis in the cornea. British J Ophthalmol. 2005;89(6):684-688.

10. Nisson MR, Dobson CM. In vitro characterization of lactoferrin aggregation and amyloid formation. Biochemistry. 2003;42(2):375-382.

11. Araki-Sasaki K, Osakabe Y, Miyata K, et al. What is this thing called "amyloidosis"? Cornea. 2009;28(suppl):80-83.

12. Sorensen CS, Rnager K, Scavenius C, et al. Fibril core of transforming growth factor beta-induced protein (TGFBIp) facilitates aggregation of corneal TGFBIp. Biochemistry. 2015;54(19):2943-2956.

13. Lakshminarayanan R, Cahurasia SS, Murugan E, et al. Biochemical properties and aggregation propensity of transforming growth factorinduced protein (TGFBIp) and the amyloid forming mutants. Ocular Surf. 2015;13(1):9-25.

14. Suesskind D, Auw-Haedrich C, Schorderet D, Munier FL, Loeffler KU. Keratoepithelin in secondary corneal amyloidosis. Graefe's Arch Clin Exp Ophthalmol. 2006;244(6):725-731.

15. Araki-Sasaki K, Hirano K, Osakabe Y, et al. Classification of secondary corneal amyloidosis and involvement of lactoferrin. Ophthalmol. 2013;120(6):1166-1172.

16. Araki-Sasaki K, Osakabe Y, Fukuoka H, Ideta R, Hirano K. Findings of secondary corneal amyloidosis with ultrahigh-resolution optical coherence tomography. Clin Ophthalmol. 2014;8:2115-2119.

17. Mercatelli R, Ratto F, Rossi F, et al. Three-dimentional mapping of the orientation of collagen corneal lamellae in healthy and keratoconic human corneas using SHG microscopy. J Biophotonics. 2017;10(1):75-83.

18. Koldso H, Andersen OJ, Nikolajsen CL, et al. Early events in the amyloid formation of the A546T mutant of transforming growth factor beta induced protein (TGFBIp) in corneal dystrophies compared to the non-fibrillating R555W and R555Q mutants. Biochemistry. 2015;54(36):5546-5556.

19. Pahuja N, Kumar NR, Shroff R, et al. Differential molecular expression of extracellular matrix and inflammatory genes at the corneal cone apex drives focal weakening in keratoconus. Invest Ophthalmol Vis Sci 2016;57(13):5372-5382.

20. Aldave AJ, Principe AH, Lin DY, Yellore VS, Small KW. Lattice dystrophy-like localized amyloidosis of the cornea secondary to trichiasis. Cornea. 2005;24(1):112-115.

21. Cortney DG, Poulsen ET, Kennedy S, et al. Protein composition of TGFBI-R124C- and TGFBI-R555W-associated aggregates suggests multiple mechanisms leading to lattice and granular corneal dystrophy. Invest Ophthalmol Vis Sci. 2015;56(8):4653-4661.
International Medical Case Reports Journal

\section{Publish your work in this journal}

The International Medical Case Reports Journal is an international, peer-reviewed open-access journal publishing original case reports from all medical specialties. Previously unpublished medical posters are also accepted relating to any area of clinical or preclinical science. Submissions should not normally exceed 2,000 words or
Dovepress

4 published pages including figures, diagrams and references. The manuscript management system is completely online and includes a very quick and fair peer-review system, which is all easy to use. Visit $\mathrm{http} / / / \mathrm{www}$. dovepress.com/testimonials.php to read real quotes from published authors. 